\title{
Isokinetic strength of fully operational U.S. Navy Seals with a previous history of shoulder and knee injury
}

\author{
Timothy C. Sell*, Nicholas C. Clark, John P. Abt, Mita Lovalekar and Scott M. Lephart \\ Warrior Human Performance Research Laboratory, University of Pittsburgh, Pittsburgh, PA, USA
}

Received 1 January 2015

Accepted 4 August 2015

\begin{abstract}
.
BACKGROUND: Unintentional musculoskeletal injury has a significant impact on military personnel which is amplified in U.S. Navy Sea, Air, and Land Operators who participate in year round physical and tactical training. Full recovery from injury including restoration of strength is necessary for safe participation in training and performance of missions. Inadequate recovery may predispose the Operator to risk of future injury.

OBJECTIVE: The purpose of this study was to examine isokinetic knee and shoulder strength of previously injured Operators who had returned to full duty.

METHODS: Two previously injured cohorts, a knee injury group $(n=46)$ and a shoulder injury group $(n=55)$, were created from a larger group of Operators $(n=305)$ who had undergone strength testing. A comparison cohort was also created from each injury group (knee injury control group $(n=77)$ and shoulder injury control group $(n=121)$. All participants underwent isokinetic strength testing of their group assigned joint. This included knee flexion/extension strength testing for the knee group and shoulder internal/external rotation strength testing for the shoulder group. Side-to-side comparisons were made within each injury group and to the control group (injured extremity to strongest extremity of the control group). Individual counts within the injured Operators with strength deficits greater than $10 \%$ in their injured extremity were also performed.

RESULTS: No significant side-to-side or between group differences were observed for the knee injury group. No significant side-to-side or between group differences were observed except for shoulder external rotation strength which was significantly different between groups ( $p=0.003$ ). Side-to-side strength deficits greater than $10 \%$ were observed in 20 to $25 \%$ of the injured Operators.

CONCLUSION: The group comparisons demonstrate the effectiveness of the military group's rehabilitation and performance training programs, but continued vigilance and tracking of injured individuals are necessary to insure full recovery and return to duty as a small number of each injured cohort did have strength deficits bilaterally.
\end{abstract}

Keywords: Injury prevention, shoulder, knee, isokinetic, strength, navy, SEALs

\section{Introduction}

${ }^{*}$ Corresponding author: Timothy C. Sell, Department of Orthopaedic Surgery, School of Medicine, Duke University, Director, Michael W. Krzyzewski Human Performance Laboratory (K-Lab), DUMC 102916, Durham, NC 27705, USA. Tel.: +1 919684 1355; E-mail: timothy.sell@duke.edu.
The prevention of unintentional musculoskeletal injury is a continued focus of human performance managers and medical personnel in the military due to the high prevalence and significant impact that injury can have on performance and tactical readiness [1-8]. In comparison to general purpose forces, injury preven- 
tion in U.S. Special Forces may have amplified importance due to the high intensity and volume of physical and tactical training as well as the high-risk missions they execute [9]. Injury prevention strategies focus on improving human capabilities and reducing modifiable risk factors for injury but the number of prospectively identified risk factors for musculoskeletal injuries remains relatively low. Previous injury is frequently cited and may be the most common prospectively determine risk factor for injury in both civilian and military populations regardless of anatomic location, type of injury, or tissue involved [10-17]. The reasoning is likely multifactorial, and the effect on functional joint stability likely plays a prominent role.

Functional joint stability can be defined as the state of a joint remaining in or promptly returning to proper alignment through the equalization of joint forces and moments [18]. This requires synergistic function between the static (ligaments, joint capsule, cartilage, friction, and bony geometry) and dynamic components of joint stability. The dynamic components include the neuromuscular control over the skeletal muscles cross the joint and are dependent on the specific skeletal muscle surrounding the joint's characteristics [18-21]. The consequences of musculoskeletal injury can involve changes in musculoskeletal tissue integrity and persistent deficits in proprioception and neuromuscular control including negative effects observed in muscular function/capability [22]. Strength, as measured by force production capability, is one of the most observable effects of injury and is justifiably targeted as part of rehabilitation prior to return to sport or return to duty [23]. Strength is essential to efficient and effective functional joint stability [24] with deficits influencing future injury [25-30]. Sapega suggested that bilateral strength deficits greater than $10 \%$ were possibly abnormal but strength deficits greater than $20 \%$ were almost certainly abnormal [31]. Schmitt and colleagues demonstrated that quadriceps strength deficits in individuals who have suffered anterior cruciate ligament injury negatively effect return to sport and result in bilateral differences in landing biomechanics [27,32]. Side-to-side strength deficits have also been prospectively associated with future injury including knee, thigh, and ankle injuries [28,33,34].

Musculoskeletal injuries in military personnel are frequent and predispose the individual to future injury. These injuries affect multiple components necessary for maintaining functional joint stability including muscle strength. Special Forces Operators participate in year round tactical and physical training in order to maintain a high level of readiness, which increases their likelihood of injury. Full recovery from injury is essential for safe performance of missions and participation in physical training. Inadequate recovery may be evident in strength deficits and could potentially predispose the Operator to future injury [35-40]. The purpose of this study was to examine isokinetic knee and shoulder strength of previously injured Operators who had returned to full duty. We hypothesized that individuals who had suffered previous knee or shoulder injury would have lower strength compared to a control group and side-to-side differences greater than ten percent. The results of this study, if our hypotheses were true, would provide evidence for the potential risk of future injury and demonstrate the need for continued monitoring of strength and appropriate physical training to resolve any persistent strength deficits.

\section{Methods}

\subsection{Participants}

All of the participants were U.S. Navy Sea, Air, and Land Operators who were part of a larger collaborative study between Naval Special Warfare and the University of the primary investigator of the current study. All testing was performed at the Warrior Human Performance Research Center located at the participants' military installation. All participants had been cleared for full duty and voluntarily consented to participate in the study. Human subject protection approval was obtained and participants provided written informed consent prior to enrollment and testing. The initial cohort of participants included 305 Operators. A total of 46 participants from this larger cohort were classified as having a unilateral knee injury (UKI) and 55 participants were classified as having a unilateral shoulder injury (USI) based on medical chart reviews. In order to be classified as part of the UKI or USI group, there had to be no medical chart history of injury to the contralateral knee for the UKI group or the shoulder for the for the USI group. An uninjured comparison group was selected for the UKI and USI groups also based on medical chart reviews. The comparison group for the UKI cohort included 77 participants and the comparison group for the USI cohort included 121 participants. The demographic data for each group are presented in Table 1.

Injury data was sourced from each of the participant's medical charts and entered in the University's 
Table 1

Participant demographics

\begin{tabular}{lcccccccc}
\hline & \multicolumn{2}{c}{ Age (years) } & & \multicolumn{2}{c}{ Height $(\mathrm{cm})$} & & \multicolumn{2}{c}{ Weight $(\mathrm{kg})$} \\
\cline { 2 - 3 } & Mean & $\pm \mathrm{SD}$ & & Mean & $\pm \mathrm{SD}$ & & Mean & $\pm \mathrm{SD}$ \\
\hline Knee injury group $(n=46)$ & 31.1 & 6.0 & & 178.2 & 5.0 & & 86.8 & 8.0 \\
Knee injury control group $(n=77)$ & 26.9 & 5.6 & & 177.6 & 6.0 & & 84.2 & 9.0 \\
Shoulder injury group $(n=55)$ & 30.6 & 6.1 & & 178.0 & 6.4 & & 87.0 & 9.4 \\
Shoulder injury control group $(n=121)$ & 27.2 & 6.1 & & 177.6 & 6.0 & & 84.5 & 8.7 \\
\hline
\end{tabular}

Table 2

List of shoulder injuries

\begin{tabular}{lc}
\hline Shoulder injury type & Count \\
\hline Rotator cuff strain & 10 \\
Labral tear & 7 \\
Subacromial impingement & 7 \\
Glenohumeral dislocation & 6 \\
Pain (unspecified) & 6 \\
Acromioclavicular joint sprain & 4 \\
Glenohumeral joint subluxation & 3 \\
Rotator cuff tendonitis & 3 \\
Acromioclavicular joint pain & 2 \\
Shoulder instability & 2 \\
Biceps tendinopathy & 1 \\
Bursitis & 1 \\
Clavicle fracture & 1 \\
Contusion & 1 \\
Scapula fracture & 1 \\
\hline
\end{tabular}

Table 3

List of knee injuries

\begin{tabular}{lc}
\hline Knee injury type & Count \\
\hline Patellar-femoral pain & 13 \\
Bursitis & 5 \\
Medial collateral ligament sprain & 5 \\
Anterior cruciate ligament sprain & 4 \\
Patellar contusion & 4 \\
Lateral collateral ligament sprain & 3 \\
Meniscal & 3 \\
Patella tendonitis & 3 \\
Contusion (location not specified) & 1 \\
Degenerative joint disease & 1 \\
Patellar dislocation & 1 \\
Osgood Schlatters disease & 1 \\
Unspecified strain & 1 \\
Unspecified pain & 1 \\
\hline
\end{tabular}

military epidemiology database [41]. The list of shoulder injuries is provided in Table 2 and the list of knee injuries is provided in Table 3. The database provides a mechanism by which injury data can be linked to laboratory testing in order for multivariate risk factor analysis to be performed. Injury data collection was performed prior to laboratory testing using the operational definitions for injury and injury type that were discussed and defined by experienced clinicians and researchers in our group and were established $a$ priori to ensure validity and consistency of the data. For purposes of this study, an injury was defined as any chart- documented history of injury to the shoulder or knee for which medical advice was sought. The comparison group of uninjured participants was defined as those participants without any chart-documented history of injury to the joint that defined the comparison group to which they were assigned. Injuries included those that could be clinically localized to the specific joint.

\subsection{Outcome measures}

Strength of the shoulder and knee were assessed with an isokinetic dynamometer (Biodex Medical Systems, Shirley, NY). Calibration of the isokinetic dynamometer was performed according to the specifications outlined in the manufacturer's service manual. Shoulder external and internal rotation was performed with participant in a seated position with the torso, waist, and thigh straps in place to reduce accessory motion and isolate shoulder external and internal rotation maximum performance. The dynamometer head was adjusted so that the shoulder was tested in a modified neutral position of approximately 15 degrees of abduction and 15 degrees of flexion. The axis-of-rotation of the dynamometer was aligned with the long axis of the humerus. Range of motion limits were set with maximum external rotation at a point just inside maximum active external rotation, and maximum internal rotation at a point just before the dynamometer shoulder attachment touched the abdomen. Reliability of the shoulder protocol has been previously established and has been demonstrated to be excellent [42]. Knee flexion and extension strength testing was performed with the participant in a seated position with the torso straps and thigh strap (for the tested lower extremity) in place in order to reduce accessory motion and isolate knee flexion and extension performance. Range of motion limits were set for knee flexion and extension with maximum flexion measured at 90 degrees and maximum extension measured at zero degrees. Reliability of the knee protocol has been previously established and has been demonstrated to be excellent [43]. The shoulder and knee strength testing protocol was a concentric-concentric reciprocal protocol with five 
Table 4

Knee strength performance

\begin{tabular}{|c|c|c|c|c|c|c|c|c|c|c|c|c|c|c|}
\hline \multirow[b]{3}{*}{ Test/variable } & \multicolumn{6}{|c|}{ Injured group } & \multirow{3}{*}{$\begin{array}{c}\text { Side-to-side } \\
\text { comparisons } \\
p \text {-value }\end{array}$} & \multicolumn{6}{|c|}{ Healthy group } & \multirow{3}{*}{$\begin{array}{c}\text { Between group } \\
\text { comparisons } \\
p \text {-value }\end{array}$} \\
\hline & \multicolumn{3}{|c|}{ Injured side } & \multicolumn{3}{|c|}{ Uninjured side } & & \multicolumn{3}{|c|}{ Right side } & \multicolumn{3}{|c|}{ Left side } & \\
\hline & Mean & $\mathrm{SD}$ & $\overline{\text { Median }}$ & Mean & $\mathrm{SD}$ & Median & & Mean & $\mathrm{SD}$ & $\overline{\text { Median }}$ & Mean & $\mathrm{SD}$ & $\overline{\text { Median }}$ & \\
\hline Knee flexion (body weights) & 1.26 & 0.28 & 1.24 & 1.30 & 0.19 & 1.32 & 0.212 & 1.32 & 0.23 & 1.28 & 1.30 & 0.23 & 1.28 & 0.235 \\
\hline Knee extension (body weights) & 2.50 & 0.50 & 2.54 & 2.59 & 0.38 & 2.55 & 0.133 & 2.65 & 0.43 & 2.71 & 2.57 & 0.46 & 2.60 & 0.073 \\
\hline
\end{tabular}

complete repetitions at $60^{\circ} / \mathrm{s}$. Prior to testing the procedures for strength testing were explained to each participant. Each participant performed three practice repetitions at 50\% maximum effort and three practice repetitions at $100 \%$ effort prior to testing for data analysis. A one-minute rest period was provided between the practice trials and the actual testing for data analysis. Testing was performed bilaterally for all participants. Average peak moment (averaged across the five repetitions) normalized to percent body weight was calculated for each of the four movements (shoulder internal/external rotation and knee flexion/extension).

\section{Statistical analysis}

The means and standard deviations were calculated for each of the strength variables. The normality of the data was assessed using the Shapiro-Wilk test. Sideto-side comparisons within each group were examined utilizing paired t-tests when data were normally distributed or with a Wilcoxon Sign Ranks test when data were not normally distributed. Between-group comparisons (injured extremity of the injury cohort compared to stronger of the two extremities of the control group cohort) were examined utilizing independent samples t-tests when data were normally distributed or with a Mann-Whitney U test when data were not normally distributed. Bilateral differences within the injury cohort were calculated and a total count of the number of individuals with side-to-side differences greater than ten percent was determined. Statistical significance was set at $p<0.05$ a priori. Statistical analyses were conducted using Stata/SE 13.0 (Statcorp, College Station, TX)

\section{Results}

Some data were normally distributed, while some were not, resulting in the use of both parametric and non-parametric tests as appropriate and indicated below as well as in the tables. The means, standard deviations, and medians for each of the variables for the unilateral knee injured group and their control group are presented in Table 4. The average knee flexion peak moment (PMf) data for the injured group data was normally distributed so paired t-tests were employed to compare PMf side-to-side. No significant side-to-side differences were observed for PMf in the injured group. The average knee extension peak moment (PMe) data for the injured group was not normally distributed so a Wilcoxon matched-pairs signedranks test was employed to compare side-to-side differences for PMe in the injured group. No significant side-to-side differences were observed for PMe in the injured group. These within group comparisons for the injured group are presented in Table 4 . Eleven of the 46 participants in injured group had a unilateral weakness exceeding $10 \%$ (compared to the uninjured extremity for PMf) with the same number regarding PMe. However, out of the eleven, only five of the participants had a simultaneous flexor and extensor weakness greater than $10 \%$.

Five individuals had unilateral weakness exceeding $20 \%$ for PMf and four had four had unilateral weakness greater than $20 \%$ for PMe. Comparisons between the injured leg of the injured group and the right leg of the control group (the stronger of the two legs) were made using an unpaired t-test for PMf and a MannWhitney U test for PMe since PMf was normally distributed but PMe was not normally distributed. Neither of these comparisons were significantly different. These comparisons are presented in Table 4.

The means, standard deviations, and medians for each of the variables for the unilateral shoulder injured group and their control group are presented in Table 5. Average shoulder external rotation peak moment (PMer) and average shoulder internal rotation peak moment (PMir) were normally distributed so paired t-tests were employed to compare side-to-side differences in the injured group and unpaired t-tests were employed to compare the injured arm of the injured group to the right arm (the stronger of the two arms) for the control group. No significant side-to-side differences were observed for PMer or PMir in the injured group. These within group comparisons for the injured group are presented in Table 5. Twelve of the 55 par- 
Table 5

Shoulder strength performance

\begin{tabular}{|c|c|c|c|c|c|c|c|c|c|c|c|c|c|c|}
\hline \multirow[b]{3}{*}{ Test/variable } & \multicolumn{6}{|c|}{ Injured group } & \multirow{3}{*}{$\begin{array}{c}\text { Side-to-side } \\
\text { comparisons } \\
p \text {-value }\end{array}$} & \multicolumn{6}{|c|}{ Healthy group } & \multirow{3}{*}{$\begin{array}{c}\text { Between group } \\
\text { comparisons } \\
p \text {-value }\end{array}$} \\
\hline & \multicolumn{3}{|c|}{ Injured side } & \multicolumn{3}{|c|}{ Uninjured side } & & \multicolumn{3}{|c|}{ Right side } & \multicolumn{3}{|c|}{ Left side } & \\
\hline & Mean & $\mathrm{SD}$ & $\overline{\text { Median }}$ & Mean & $\mathrm{SD}$ & Median & & Mean & SD & Median & Mean & $\mathrm{SD}$ & $\overline{\text { Median }}$ & \\
\hline External rotation (body weights) & 0.43 & 0.09 & 0.43 & 0.42 & 0.08 & 0.44 & 0.618 & 0.47 & 0.08 & 0.48 & 0.43 & 0.07 & 0.43 & 0.003 \\
\hline Internal rotation (body weights) & 0.68 & 0.20 & 0.67 & 0.68 & 0.18 & 0.66 & 0.937 & 0.73 & 0.16 & 0.73 & 0.71 & 0.17 & 0.70 & 0.108 \\
\hline
\end{tabular}

ticipants in injured group had a unilateral weakness exceeding 10\% (compared to the uninjured extremity for PMer) and likewise eleven had such a weakness for PMir. Four of the participants had deficits greater than $10 \%$ for both shoulder movements tested. Three individuals had unilateral weakness exceeding $20 \%$ for PMer and three individuals had similar weakness for PMir. Comparisons between the injured arm of the injured group and the right arm of the control group demonstrated significant differences for PMer (control group stronger) but not PMir. These comparisons are presented in Table 5.

\section{Discussion}

Unintentional musculoskeletal injury compromises functional joint stability. Inadequate rehabilitation and recovery can reduce the individual operator's performance and tactical readiness. The purpose of this study was to examine knee and shoulder strength of individuals with a previous history of injury and determine if deficits in strength persisted despite return to full duty. We hypothesized that individuals who had suffered a previous injury would demonstrate significant deficits in strength at the time of testing. The majority of the results did not support our hypotheses, which demonstrates the positive effect that rehabilitation and performance teams are having on returning the Operators to deployment eligibility status. The only result that did support our hypothesis was shoulder external rotation strength compared between groups. While the majority of individuals have successfully rehabilitated based on these results, these comparisons should be interpreted cautiously as approximately $20 \%$ to $25 \%$ of those Operators who did have a previous injury had side-toside strength differences that indicated a greater than ten percent deficit in strength compared to their uninjured side. Overall, the results demonstrate the success of the group's rehabilitation and performance training programs, but they also indicate the need for continued vigilance and testing of individuals with previous injury to insure that full recovery, at least based on strength, has occurred.
Unintentional musculoskeletal injury is a significant concern for military personnel and is a greater concern in U.S. Special Forces [9]. United States Special Forces must maintain a high level of readiness that dictates year round physical training. The majority of musculoskeletal injuries occur during physical training so year round physical training results in a high incidence of unintentional musculoskeletal injury $[9,41]$. Special Forces Operators suffer a wider distribution of injuries across multiple joints including the shoulder, spine, knee, and ankle then general purpose forces $[9,41]$. The higher incidence and greater distribution of injuries across multiple joints stresses rehabilitation and performance training personnel and demonstrates to continued requirement to identify risk factors for injury. The identification of risk factors for musculoskeletal injuries is an ongoing research initiative across many different organizations. A commonly identified risk factor for future injury is a history of previous injury [10-17]. Injury compromises tissue integrity and impairs functional joint stability [22]. A lack of full recovery from injury can compromise performance and may lead to future injury, which may be due to persistent deficits in strength and the subsequent effects on functional joint stability.

The maintenance of functional joint stability in the presence of destabilizing forces requires a complex interaction between multiple systems [18]. Strength is one aspect of the dynamic components of joint stability, and is frequently studied following injury, surgery, rehabilitation and return to play. For comparison purposes, the shoulder and knee strength of the injured and uninjured cohorts in the current study were greater than infantry soldiers and highly trained athletes (triathletes) based on data collection using identical procedures in an independent study [41]. This would indicate that the current group of subjects is also highly trained. Injuries to the knee and shoulder can compromise functional joint stability characteristics such as strength and can delay a return to sport and can result in subsequent or recurrent injury [25-30]. Forty-six individuals in the current study had a medical chart documented history of unilateral knee injury. No significant differences were observed side-to-side in this co- 
hort and as a group their strength was not significantly different to the uninjured comparison cohort. In order to examine the effects of injury on the individual an examination of subject specific data was also performed. Overall, $76 \%$ of the individuals demonstrated side-toside strength that was within $10 \%$ of the uninjured knee leaving $24 \%$ of individuals demonstrating continued deficits greater than $10 \%$, of whom 5 individuals had deficits greater than $20 \%$ bilateral difference for knee flexion strength and 4 individuals had similar deficits for knee extension strength. The latter is considered pathological and requires specific attention [31]. It is not unusual to see persistent deficits in strength following injury. For example, previous injury to the meniscus, the hamstrings musculature, and the anterior cruciate ligament (repaired or conservatively managed) results in persistence strength deficits in a significant percentage of individuals [35-37]. Stensrud et al. examined individuals with degenerative meniscal tears and demonstrated significant side-to-side deficits in quadriceps strength and with half of the group demonstrating significant "clinically relevant impairments" (greater than 10\%) [35]. The percentage of individuals with deficits in the Stensrud et al. analysis is twice as high as the current study. Similar results have been observed following anterior cruciate ligament injury (ACL) [36]. In a two year follow-up in a cohort of ACL injured individuals, Grindem et al. demonstrated that many individuals ( $23 \%$ to $35 \%$ ) continued to have knee flexion and extension strength deficits with percentages of individuals with deficits similar to the current study or higher. O'Sullivan and colleagues assessed isokinetic strength in a group of Gaelic footballers who had previously suffered hamstring injuries [37]. The Gaelic footballers' previous injured extremity had significantly lower hamstrings/quadriceps strength ratios and weaker hamstrings when matched for limb dominance. The percentage of individuals with strength deficits greater than ten percent was not reported. The knee injury cohort in the current study who had a history of previous knee injury demonstrated as a group that they do not have bilateral deficits in knee strength and they do not have strength deficits compared to an uninjured control group; although, the side-to-side deficits in the low percentage of individuals should warrant ongoing attention.

The results of the shoulder injury cohort comparison differed slightly from the knee injury cohort. In the current study no significant difference were observed side-to-side in the injured group, but a significant difference was observed between groups for shoulder ex- ternal rotation strength with the control group being stronger. The examination of subject specific strength deficits revealed that approximately $20 \%$ of the injured group had side-to-side differences greater than $10 \%$ for shoulder external rotation strength and internal rotation strength. Furthermore, 6 individuals had strength deficits greater than 20\% [31]. The effect of previous injury on strength at the shoulder has also been observed in other groups of individuals with a previous history of shoulder injury [38-40]. Erol and colleagues examined a cohort of individuals who all had either stage I or stage II subacromial impingement syndrome [39]. Overall, isokinetic strength testing showed no significant strength differences between limbs in the injured cohort and no significant strength differences between the injured cohort and a control group. But, there was a significant side-to-side difference in internal rotation peak moment compared to the side-toside difference in the control group. Meller and colleagues also observed strength deficits when they examined a cohort of participants who had recurrent anterior shoulder instability who had undergone a stabilization procedure [40]. There was a significant difference in external rotation strength between the injured and uninjured shoulder although five of the 27 participants did not demonstrate side-to-side differences. Edouard and colleagues also examined a group of individuals with recurrent glenohumeral instability [38]. Edouard and colleagues tested both an injured group and a control group at multiple velocities. Overall, they concluded that individuals with recurrent glenohumeral shoulder instability had weaker internal and external rotators although the differences were different in magnitude depending on whether the injured extremity was the dominant or non-dominant limb.

There are some potential limitations to the current study. The data describing the injuries in the medical records is limited. In some instances the description only indicates the major joint involved (knee for example) and that a "strain" occurred. A more detailed description of the injury that included days lost or surgical intervention would have provided greater insight into the severity of the injury and potential reason for persistent deficits observed bilaterally for strength.

\section{Conclusion}

The primary outcome of this study is that as a group, individuals who have suffered a previous knee or shoulder injury have returned to strength levels sim- 
ilar to their uninjured side and are similar to an uninjured control group (with the exception of shoulder external rotation strength). These group comparisons demonstrate the effectiveness of the military group's rehabilitation and performance training programs, but continued vigilance and tracking of injured individuals are necessary to insure full recovery and return to duty as a small number of each injured cohort did have bilateral strength deficits greater than $10 \%$. There are potential limitations to the current study including the type of injury suffered and the severity of injury. Typically, strength comparisons in injured groups attempt to group similar injuries for comparison. While the different injury types included in each of the cohorts may confound the results of the statistical analysis, the ability to screen for potential strength deficits based on whether a joint is injured regardless of type of injury adds value to screening capabilities. From a rehabilitation perspective, if an individual presents with clinically significant side-to-side differences in muscle strength, then it is clinically important to administer muscle strength training interventions, regardless of the exact type of injury that may have preceded the muscle strength deficit. In summary, medical chart documented history of injury should be utilized as a screening factor for ongoing monitoring of strength to insure that individualized training matches injury rehabilitation and prevention requirements.

\section{Acknowledgments}

This work was supported by the Office of Naval Research, Grant \#N00014071190/N000140810412.

\section{Conflict of interest}

The authors declare no conflict of interest.

\section{References}

[1] Garamone J. Reducing Sports Injuries http: //www.defense. gov2001, [updated Mar 27; cited 2015 April 20, 2015]. Available from: http: //www.defense.gov/news/newsarticle.aspx?id $=457 \mathrm{ww} 53$.

[2] Jones BH, Perrotta DM, Canham-Chervak ML, Nee MA, Brundage JF. Injuries in the military: a review and commentary focused on prevention. American Journal of Preventive Medicine. 2000; 18(3): 71-84.

[3] Kelley PW, United States. Dept. of the Army. Office of the Surgeon General. Military preventive medicine: mobilization and deployment. Washington, D.C.: Borden Institute, Walter Reed Army Medical Center; 2003.
[4] Lauder TD, Baker SP, Smith GS, Lincoln AE. Sports and physical training injury hospitalizations in the army. American Journal of Preventive Medicine. 2000; 18(3): 118-28.

[5] Litow FK, Krahl PL. Public health potential of a disability tracking system: Analysis of U.S. navy and marine corps physical evaluation boards 2005-2006. Military Medicine. 2007; 12: 1270-4.

[6] Popovich RM, Gardner JW, Potter R, Knapik JJ, Jones BH. Effect of rest from running on overuse injuries in army basic training. American Journal of Preventive Medicine. 2000; 18(3): 147-55.

[7] Smith GS, Dannenberg AL, Amoroso PJ. Hospitalization due to injuries in the military. Evaluation of current data and recommendations on their use for injury prevention. American Journal of Preventive Medicine. 2000; 18(3): 41-53.

[8] Songer TJ, LaPorte RE. Disabilities due to injury in the military. American Journal of Preventive Medicine. 2000; 18(3): 33-40.

[9] Abt JP, Sell TC, Lovalekar MT, Keenan KA, Bozich AJ, Morgan JS, et al. Injury epidemiology of U.S. army special operations forces. Mil Med. 2014; 179(10): 1106-12.

[10] Cameron KL, Mountcastle SB, Nelson BJ, DeBerardino TM, Duffey ML, Svoboda SJ, et al. History of shoulder instability and subsequent injury during four years of follow-up: A survival analysis. J Bone Joint Surg Am. 2013; 95(5): 439-45.

[11] Greene HS, Cholewicki J, Galloway MT, Nguyen CV, Radebold A. A history of low back injury is a risk factor for recurrent back injuries in varsity athletes. Am J Sports Med. 2001; 29(6): 795-800.

[12] Hagglund M, Walden M, Ekstrand J. Risk factors for lower extremity muscle injury in professional soccer: The UEFA injury study. Am J Sports Med. 2013; 41(2): 327-35.

[13] Hill OT, Kay AB, Wahi MM, McKinnon CJ, Bulathsinhala L, Haley TF. Rates of knee injury in the U.S. active duty army, 2000-2005. Mil Med. 2012; 177(7): 840-4.

[14] Nilstad A, Andersen TE, Bahr R, Holme I, Steffen K. Risk factors for lower extremity injuries in elite female soccer players. Am J Sports Med. 2014; 42(4): 940-8.

[15] Richmond SA, Fukuchi RK, Ezzat A, Schneider K, Schneider G, Emery CA. Are joint injury, sport activity, physical activity, obesity, or occupational activities predictors for osteoarthritis? A systematic review. J Orthop Sports Phys Ther. 2013; 43(8): 515-B19.

[16] Robinson CM, Seah M, Akhtar MA. The epidemiology, risk of recurrence, and functional outcome after an acute traumatic posterior dislocation of the shoulder. J Bone Joint Surg Am. 2011; 93(17): 1605-13.

[17] Wilkinson DM, Blacker SD, Richmond VL, Horner FE, Rayson MP, Spiess A, et al. Injuries and injury risk factors among British army infantry soldiers during predeployment training. Inj Prev. 2011; 17(6): 381-7.

[18] Riemann BL, Lephart SM. The sensorimotor system, Part I. The physiologic basis of functional joint stability. Journal of Athletic Training. 2002; 37(1): 71-9.

[19] Solomonow M, Krogsgaard M. Sensorimotor control of knee stability: A review. Scand J Med Sci Sports. 2001; 11(2): 6480.

[20] Johansson H, Sjolander P. The neurophysiology of joints. In: Wright V, Radin EL, editors. Mechanics of Joints: Physiology, Pathophysiology, and Treatment. New York, NY: Marcel Dekker Inc.; 1993; p. 243-90.

[21] Lew WD, Lewis JL, Craig EV. Stabilization by capsule, ligaments, and labrum: stability at the extremes of motion. In: Matsen FA, Fu FH, Hawkins RJ, editors. The Shoulder: A 
Balance of Mobility and Stability. Rosemont, IL: American Academy of Orthopaedic Surgeons; 1993; p. 69-89.

[22] Lephart SM, Henry TJ. The Physiological Basis for Open and Closed Kinetic Chain Rehabilitation for the Upper Extremity. Journal of Sport Rehabilitation. 1996; 5(1): 71-87.

[23] Swanik CB, Lephart SM, Giannantonio FP, Fu FH. Reestablishing Proprioception and Neuromuscular Control in the ACL-Injured Athlete. Journal of Sport Rehabilitation. 1997; 6: $182-206$

[24] Nagai T, Sell TC, House AJ, Abt JP, Lephart SM. Knee proprioception and strength are correlated with landing kinematics during a single-leg stop-jump task. Journal of Athletic Training. (In Press).

[25] Croisier JL, Ganteaume S, Binet J, Genty M, Ferret JM. Strength imbalances and prevention of hamstring injury in professional soccer players: a prospective study. Am J Sports Med. 2008; 36(8): 1469-75.

[26] Edouard P, Degache F, Oullion R, Plessis JY, Gleizes-Cervera $\mathrm{S}$, Calmels P. Shoulder strength imbalances as injury risk in handball. Int J Sports Med. 2013; 34(7): 654-60.

[27] Schmitt LC, Paterno MV, Hewett TE. The impact of quadriceps femoris strength asymmetry on functional performance at return to sport following anterior cruciate ligament reconstruction. J Orthop Sports Phys Ther. 2012; 42(9): 750-9.

[28] Sugiura Y, Saito T, Sakuraba K, Sakuma K, Suzuki E. Strength deficits identified with concentric action of the hip extensors and eccentric action of the hamstrings predispose to hamstring injury in elite sprinters. J Orthop Sports Phys Ther. 2008; 38(8): 457-64.

[29] Myer GD, Ford KR, Barber Foss KD, Liu C, Nick TG, Hewett TE. The relationship of hamstrings and quadriceps strength to anterior cruciate ligament injury in female athletes. Clin $\mathbf{J}$ Sport Med. 2009; 19(1): 3-8.

[30] Baumhauer JF, Alosa DM, Renstrom AF, Trevino S, Beynnon B. A prospective study of ankle injury risk factors. Am Sports Med. 1995; 23(5): 564-70.

[31] Sapega AA. Muscle performance evaluation in orthopaedic practice. J Bone Joint Surg Am. 1990; 72(10): 1562-74.

[32] Schmitt LC, Paterno MV, Ford KR, Myer GD, Hewett TE. Strength Asymmetry and Landing Mechanics at Return to Sport after Anterior Cruciate Ligament Reconstruction. Med Sci Sports Exerc. 2015; 47(7): 1426-34.

[33] Boling MC, Padua DA, Marshall SW, Guskiewicz K, Pyne S, Beutler A. A prospective investigation of biomechanical risk factors for patellofemoral pain syndrome: the Joint Undertaking to Monitor and Prevent ACL Injury (JUMP-ACL) cohort. Am J Sports Med. 2009; 37(11): 2108-16.

[34] Leetun DT, Ireland ML, Willson JD, Ballantyne BT, Davis IM. Core stability measures as risk factors for lower extremity injury in athletes. Med Sci Sports Exerc. 2004; 36(6): 926-34.

[35] Stensrud S, Risberg MA, Roos EM. Knee function and knee muscle strength in middle-aged patients with degenerative meniscal tears eligible for arthroscopic partial meniscectomy. Br J Sports Med. 2014; 48(9): 784-8.

[36] Grindem H, Eitzen I, Engebretsen L, Snyder-Mackler L, Risberg MA. Nonsurgical or Surgical Treatment of ACL Injuries: Knee Function, Sports Participation, and Knee Reinjury: The Delaware-Oslo ACL Cohort Study. J Bone Joint Surg Am. 2014; 96(15): 1233-41.

[37] O'Sullivan K, O'Ceallaigh B, O'Connell K, Shafat A. The relationship between previous hamstring injury and the concentric isokinetic knee muscle strength of Irish Gaelic footballers. BMC Musculoskelet Disord. 2008; 9: 30.

[38] Edouard P, Degache F, Beguin L, Samozino P, Gresta G, Fayolle-Minon I, et al. Rotator cuff strength in recurrent anterior shoulder instability. J Bone Joint Surg Am. 2011; 93(8): 759-65.

[39] Erol O, Ozcakar L, Celiker R. Shoulder rotator strength in patients with stage I-II subacromial impingement: relationship to pain, disability, and quality of life. J Shoulder Elbow Surg. 2008; 17(6): 893-7.

[40] Meller R, Krettek C, Gosling T, Wahling K, Jagodzinski $\mathrm{M}$, Zeichen J. Recurrent shoulder instability among athletes: changes in quality of life, sports activity, and muscle function following open repair. Knee Surg Sports Traumatol Arthrosc. 2007; 15(3): 295-304.

[41] Sell TC, Abt JP, Crawford K, Lovalekar M, Nagai T, Deluzio $\mathrm{JB}$, et al. Warrior Model for Human Performance and Injury Prevention: Eagle Tactical Athlete Program (ETAP) - Part I. Journal of Special Operations Medicine. 2010; 10(4): 2-21.

[42] Sell TC, Tsai YS, Smoliga JM, Myers JB, Lephart SM Strength, flexibility, and balance characteristics of highly proficient golfers. J Strength Cond Res. 2007; 21(4): 1166-71.

[43] Abt JP, Sell TC, Laudner KG, McCrory JL, Loucks TL, Berga SL, et al. Neuromuscular and biomechanical characteristics do not vary across the menstrual cycle. Knee Surg Sports Traumatol Arthrosc. 2007; 15(7): 901-7. 